\title{
La lucha: una parte integral de las artes de combate en Irán
}

\author{
Manouchehr Moshtagh Khorasani
}

Investigador independiente (Alemania)

\author{
Recibido 14/09/2010 - Aceptado 06/10/2010
}

\section{Resumen}

La lucha tuvo y sigue teniendo un papel muy importante en la cultura marcial iraní. El presente artículo discute en primer lugar los términos y diferentes usos de la lucha, ya fuese en duelos a brazo partido, esto es, sin armas, o en combinación con armas. Seguidamente se analizan tres documentos relevantes, como son un manuscrito sobre la lucha anónimo y sin título del periodo de Šāh Esmāil Safávida (1502-1524 d.C.), el manuscrito Tumār-e Puryāye Vali, también anónimo y probablemente escrito durante el periodo sáfavida (1502-1722 d.C.), y la obra del famoso poeta Mirnejāt Masnavi-ye Golkošti-ye Mirnejāt, de finales del periodo de Šāh Soleymān Safávida (1666-1694 d.C.) o principios del periodo de Šāh Soltān Hosseyn Safávida (1694-1722 d.C.). A partir de las denominaciones y descripciones de las técnicas que realizan algunos de estos manuscritos, es posible apreciar cómo las formas de lucha tradicionales de Irán han conservado algunas de estas técnicas.

Palabras clave: Artes marciales, artes marciales persas, técnicas, manuscritos, batalla.

\section{Wrestling: An Integral Part of Iranian Fighting Arts}

Abstract: Wrestling played and has been playing a very important role in Iranian cultural heritage. First, the present article discusses the terms and different uses of wrestling techniques be it in unarmed duels or in combination with weapons. Then, three relevant manuscripts will be analyzed such as an untitled wrestling manuscript by an anonymous author from the period of Šāh Esmāil Safavid (1502-1524 C.E.), the manuscript Tumār-e Puryā-ye Vali also written by an anonymous author during the Safavid period (1502-1722 C.E.) and the work by a famous poet named Mirnejāt with the title Masnavi-ye Golkošti-ye Mirnejāt that was written towards the end of the period of Šāh Soleymān Safavid (16661694 C.E.) or the beginning of the period of Šāh Soltān Hosseyn Safavid (1694-1722 C.E.). Based on some terms and descriptions of the techniques found in these manuscripts, it is possible to appreciate how traditional forms of wrestling in Iran have preserved some of these techniques.

Key words: Martial Arts, Persian Martial Arts, techniques, manuscripts, battle.

\section{A luta: uma parte integral das artes de combate do Irão}

Resumo: A luta teve e continuar a ter um papel muito importante na cultura marcial iraniana. O presente artigo discute, em primeiro lugar, os termos e os diferentes usos da luta, duelos com o braço partido, isto é, sem armas, ou em combinação com armas. Seguidamente, analisam-se três documentos relevantes, como são o manuscrito, anónimo e sem título, sobre a luta do período de Šāh Esmāil Safavid (1502-1524 d.C.), o manuscrito Tumār-e Puryāye Vali, também anónimo e provavelmente escrito durante o período sáfavida (1502-1722 d.C.), e a obra do famoso poeta Mirnejāt Masnavi-ye Golkošti-ye Mirnejāt, dos finais do

Todas las fotografías son cortesía del autor, excepto cuando se señale lo contrario. 
ESCENA DE BATALLA DE

UN MANUSCRITO DE

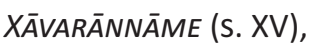
TITULADO “MĀLLIK ESTÁ LUCHANDO CON ŠĀPUR YALI", MUESTRA CÓMO MĀLLIK LANZA A SU OPONENTE.

Cortesía de Ministry of Culture and Islamic

Guidance. período de Šāh Soleymān Safávida (1666-1694 d.C.) ou princípios do período de Šāh Soltān Hosseyn Safávida (1694-1722 d.C.). A partir das denominações e descrições das técnicas, que surgem em alguns destes manuscritos, é possível apreciar como as formas de luta tradicionais do Irão conservaram algumas destas técnicas.

Palavras-chave: Artes marciais, artes marciais persas, técnicas, manuscritos, batalha.

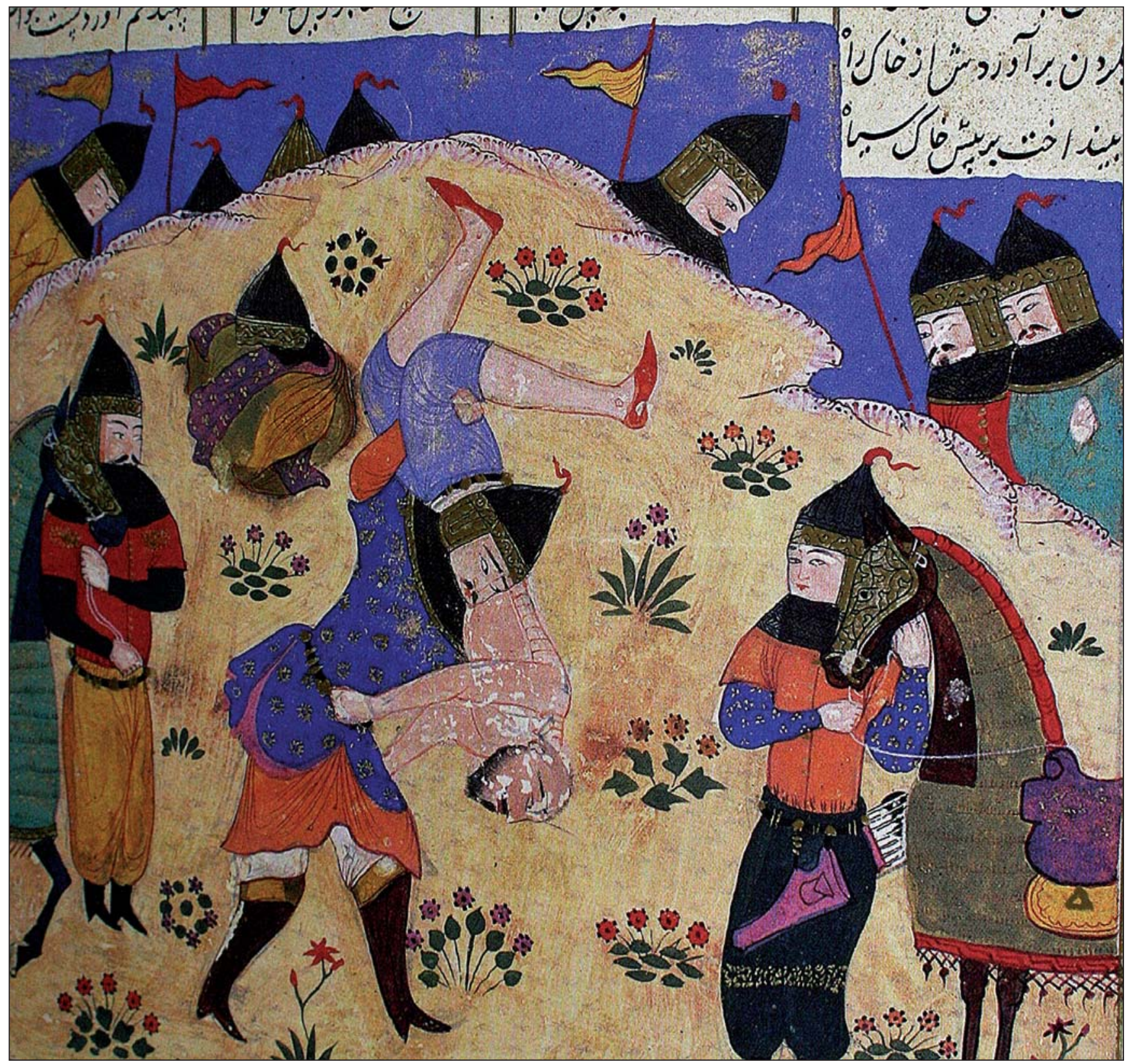




\section{La lucha: una parte integral de las artes de combate en Irán}

Manouchehr Moshtagh Khorasani

Investigador independiente (Alemania)

\section{Introducción}

Como en muchas otras culturas, la lucha jugó un papel importante en la cultura marcial iraní, hayándose representaciones del arte de la lucha talladas en fuentes de plata doradas del Imperio Sasánida (226-651 d.C.). Los manuscritos y epopeyas persas relatan que antes de una batalla los campeones de ambos ejércitos se desafiaban a un duelo. Este duelo tenía tres partes, en la que se utilizaban tres armas diferentes. Si no se lograba derrotar al oponente, entonces se utilizaba la lucha para asegurar la victoria. Cuando uno de los guerreros lograba derribar al suelo a su oponente y sentarse sobre su pecho o abdomen mostrando su dominio, podía sacar su daga o cuchillo para rematarlo.

Los campeonatos de lucha también han sido una constante histórica, celebrándose cada año un campeonato de lucha para determinar el campeón del país, que se llamaba jahānpahlavān (el campeón del mundo). Cada rey tenía un grupo de campeones de lucha en su corte (Afšāri, 2003/1382:73), que además solían ostentar otras importantes responsabilidades, por ejemplo vigilar las ciudades durante las noches (Kāzemini, 1964/1343:65; Dāstān-e Hosseyn Kord-e Šabestari, 2003/1382:30).

Con pocos altibajos, la popularidad de la lucha se ha mantenido hasta finales del Periodo Qājār (1794-1925 d.C.) (Kāzemini, 1964/1343:45). Esto se aprecia claramente a través de los manuscritos, en los que la lucha es una presencia constante. Así, por ejemplo, en el Bustān, del s. XIII (Sa'di, 2005/1383:185) se resalta su importancia con la frase "Con luchar, cazar y jugar al polo, un hombre llega a ser un guerrero".

En el Persa Nuevo la lucha se denomina košti o kosti (Ferdŏsi, 1995/1384:208; Qatrān-e Tabrizi, 1983/1362:422)1. Esta palabra tiene su raíz en el término del Persa Medio kustik (Farahvaši, 2002b/1381b:408), que significa "cinturón sagrado", aunque Zākeri (1995:75) señala que la tradición de portar el kustik podría remontarse al Antiguo Irán. Kustik también expresaba la relación entre el rey y sus súbditos, y los zoroástricos portaban un cinturón llamado kustik como símbolo de obediencia y fiel pertenencia a su religión. De hecho, la palabra kustik aparece a menudo en el Avestā con el significado de "cinturón" o

1 Véase también Asadi Tusi (1938/1317: 396), Mobārak Šāh Faxr-e Modabbar (1967/1346: 468), Molavi (2006/1385: 435), Hakim (s.f.: 585), Romuz-e Hamze (1940/1359 Hégira: 1725), y Afif (2007/1385: 367). 
MiniatURA DE UN MANUSCRITO DEL ŠĀHNĀME DEL PERIODO SAFÁVIDA, MUESTRA A UN DEMONIO TRATANDO DE SUJETAR SIN ÉXITO LA MANO DERECHA DE SU ADVERSARIO, QUE PORTA UNA ESPADA. AUNQUE SE TRATA DE UNA LEYENDA, MUCHOS RELATOS HISTÓRICOS SEÑALAN QUE ESTA ERA UNA ACCIÓN COMÚN DURANTE LAS BATALLAS.

Cortesía de Rezā Abbāāi Museum.

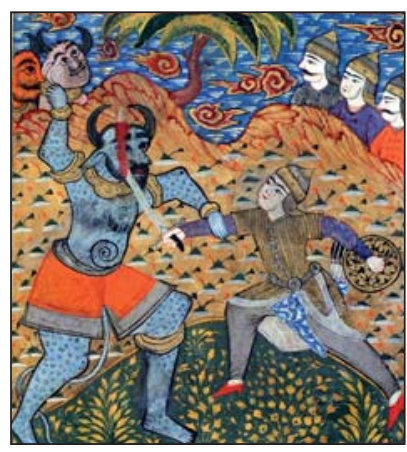
llas que servían para sujetar la mano de un oponente que llevaba un arma (como la espada) y contraatacar con la propia arma o derribarlo. Tal es el caso de las técnicas band-e dast rā gereftan va šamšir rā gardānidan va u rā bardāštan va bar zamin zadan (sujetar la muñeca de la mano [del enemigo que porta la espada] y girar la espada [mano], levantarlo [al enemigo], y soltarlo sobre el suelo) (Romuz-e Hamze, 1940/1359 de la Hégira: 42) y band-e dast rā gereftan va tiq bedar āvardan va yek dast garibān va yek dast kamar rā gereftan va ruy-e dast āvardan va bar zamin zadan (sujetar la muñeca de la espada [del enemigo], desarmar la espada, sujetar el collarín de la armadura con una mano y sujetar la espalda [del enemigo], y soltarlo sobre el suelo) (Romuz-e Hamze, 1940/1359 de la Hégira: 98).

En segundo lugar, estaban las técnicas propias de la lucha a brazo partido, que se aplicaban en un enfrentamiento sin armas cuando los guerreros las habían perdido o destrozado, o cuando dos guerreros de los ejércitos enemistados hacían un duelo de campeones antes de la batalla principal. También aquí podemos distinguir dos tipos de técnicas, según se aplicasen a caballo o a pie. Las técnicas a caballo se basaban fundamentalmente en sujetar el cinturón, la mano u otra parte del cuerpo o armadura del enemigo para descabalgarlo, como describen las técnicas ze zin dar robudan (robar de la silla) (Asadi Tusi, 1938:1317:48), qovvat kardan (poner la fuerza), en la que los guerreros agarraban el cinturón del oponente y "ponían fuerza" para desmontarlo (al-Kāteb al-Rajāni, 2004/1383:1283), garibān rā gereftan va bar bālāye sar bordan va bar zamin zadan (tomar el collarín, levantar [al enemigo] sobre la cabeza y soltarlo sobre el suelo) (Šabānkāre'i, 2002/1381:304) y kamarband gereftan va az

2 Véase también Beyqami (2002/1381: 877; vol.1) y Xonji Esfahāni (2003/1382: 81).

ce años cada zoroástrico se ata el kustik tres veces sobre la cintura, simbolizando tres principios fundamentales de su religión: "buenos pensamientos", "buenas palabras" y "buenas acciones".

Existen semejanzas entre el cinturón utilizado por la caballería (fotovvat) del periodo islámico, denominado shadd, y el kustik zoroástrico (Zakeri, 1995:309). En la ceremonia de iniciación de la fotovvat, se ataba el shadd sobre la cintura del aspirante tres o cuatro veces, simbolizando que había obtenido el suficiente nivel de madurez y superado los principios y técnicas de la organización. Los ayyārān (un grupo de guerreros) usaban un proceso de iniciación similar denominado marāsem-e oryāni (celebración desnuda), ya que se realizaba vistiendo únicamente un pantalón (Aštari Tafreši, 2000/1379:35).

En Persa Nuevo el luchar se denomina košti gereftan [literalmente "tomar lucha"] (Mobārak Šāh Faxr-e Modabbar, 1967/1346:468), y koštigir designa al luchador (Rašidoldin Fazlollāh Hamedāni, 1985/1374: 498). El campeón recibe la denominación de pahlavān (Daqiqi Tusi, 1994/1373: 78)².

\section{Técnicas de la lucha en batalla}

Diversos manuscritos persas destacan la importancia de la lucha durante la batalla. Según las descripciones que ofrecen estos manuscritos, puede deducirse la existencia de dos tipologías de técnicas de lucha. En primer lugar, aque- 


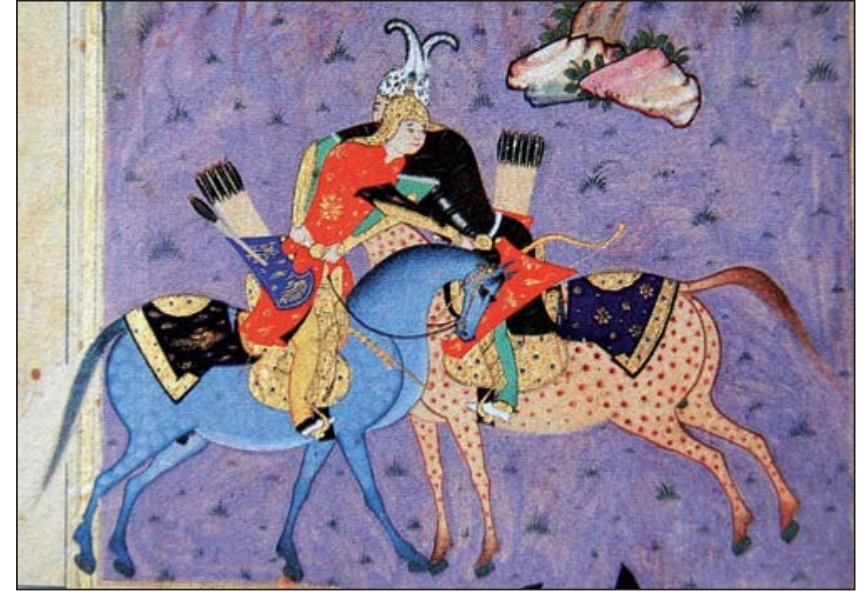

“LA BATALlA ENTRE ROSTAM Y SOHRĀB" DEL ŠĀHNĀME-YE ŠĀH ESMĀILI (PERIODO SAFÁVIDA), MUESTRA A AMBOS GUERREROS TRATANDO DE DESCABALGAR A SU OPONENTE CON LA TÉCNICA "PONER FUERZA".

Cortesía de Rezā Abbāsi Museum.

pošt-e zin boland kardan (sujetar el cinturón y levantar de la silla [del caballo]) (al-Kāteb al-Rajāni, 2004/1383:229).

Por su parte, el segundo grupo de técnicas se aplicaba de pie, como la técnica davāl-e kamar rā gereftan (sujetar el cinturón), kalle bar kalle nahādan (poner la cabeza sobre la cabeza [del oponente]), garibān rā gereftan (sujetar el collarín de la armadura [del enemigo]), garibān rā gereftan va bar zamin zadan (sujetar el collarín de la armadura [del enemigo] y soltarlo sobre el suelo), garibān rā gereftan va bar zamin zadan va bar sine nešastan (sujetar el collarín de la armadura [del enemigo], soltarlo sobre el suelo y sentarse sobre su pecho), y panje dar panje afkandan (poner la palma en la palma [del enemigo]). La lucha persa también contenía una serie de golpes como kalle bar kalle zadan o kallekub (golpear la cabeza contra la cabeza) (Romuz-e Hamze, 1940/1359 de la Hégira; Širuye Nāmdār, 2005/1384; Majma' al-Ansāb, Šabānkāre'i, 2002/1381; Masnavi Gol Košti, Abbāsi, 1995:175).

Mobārak Šāh Faxr-e Modabbar (1967/1346:468-469) explica que cuando tras la lucha con armas no había resultado un vencedor, y cuando los caballos habían muerto, se utilizaba la lucha para obtener la victoria. La primera técnica solía ser band-e dast gereftan va pičāndan (sujetar la muñeca y girar el brazo). Si esta técnica no tenían éxito, los guerreros trataban de sujetar el cuello del rival y girarlo desde arriba, como describe la técnica dast dar gardan-e yekdigar andāxtan va be alm gardan pičāndan (sujetar el cuello y girarlo desde arriba). Si esta técnica tampoco resultaba, los 


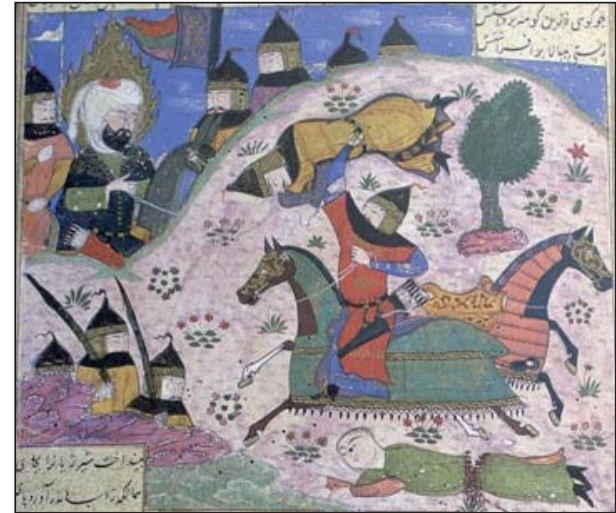

ESCENA DE UN MANUSCRITO DE XĀVARĀNNĀME

(S. XV), TITULADA “EMĀM ALI OBSERVA A MĀLEK EN LA BATALLA”. MĀLEK ESTÁ LANZANDO A SU OPONENTE TRAS APLICAR UNA TÉCNICA DE LUCHA A CABALLO.

\section{Cortesía de Ministry of Culture and Islamic}

Guidance.

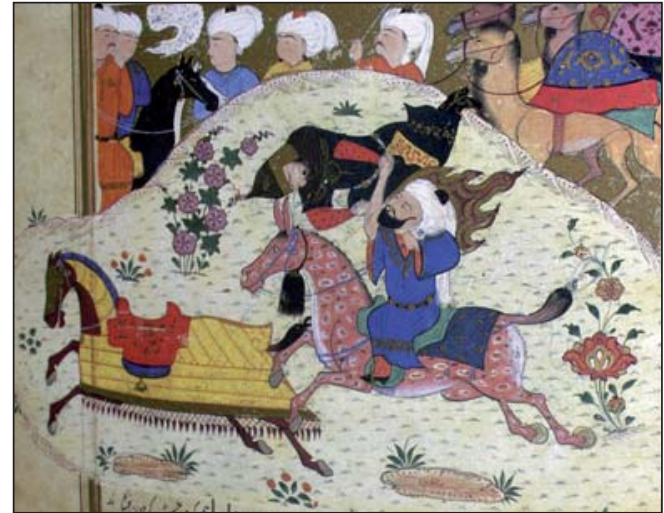

ESCENA DE UN MANUSCRITO DEL XĀVARĀNNĀME (S. XV), TITULADA “EMĀM ALI EN BATALLA CONTRA MIR SAYYĀF”. EMĀM ALI LANZA A SU OPONENTE TRAS APLICAR UNA TÉCNICA DE LUCHA A CABALLO.

Cortesía de Ministry of Culture and Islamic Guidance.

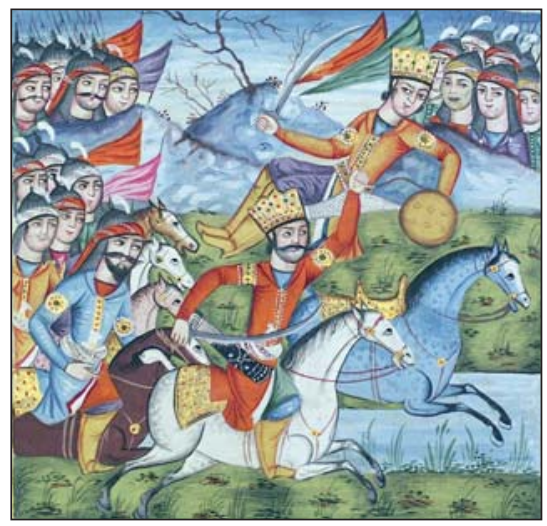

MINIATURA DE UN MANUSCRITO DEL NEGĀRESTĀN, EN LA QUE SE APRECIA UNA ACCIÓN SIMILAR.

Cortesía de Rezā Abbāsi Museum.

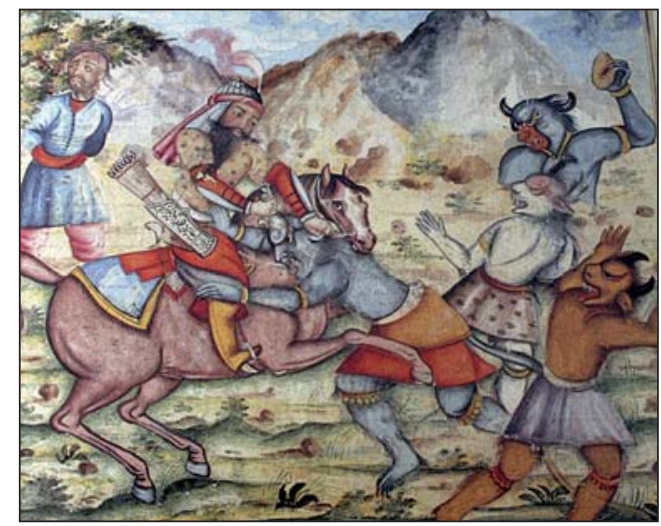

MiNIATURA DE UN MANUSCRITO DEL ŠĀHNĀME (S. XI DE LA HÉGIRA) TITUladA "Rostam ESTÁ MATANDO A ARĴANG DIV”, EN LA QUE EL DEMONIO TRATA DE DESCABALGAR A ROSTAM.

Cortesía de National Library of Iran.

guerreros intentaban sujetar las piernas con las manos y levantar al rival (dast bepāy va sāq-e yekdigar andāxtan va zur kardan), o levantarlo agarrando el cinturón (kamar-e yekdiggar gereftan va govvat kardan). Según este autor el objetivo era sujetar al enemigo y lanzarlo sobre el suelo, como describe la técnica mard rā robudan va bar zamin zadan (robar el hombre [enemigo] y lanzarlo sobre el suelo). Si todas estas técnicas eran infructuosas, el guerrero tenía que hacer la zancadilla al oponente para derribarlo, como explica la técnica paspāy zadan (golpear [con la pierna] detrás de la pierna [del oponente]). 


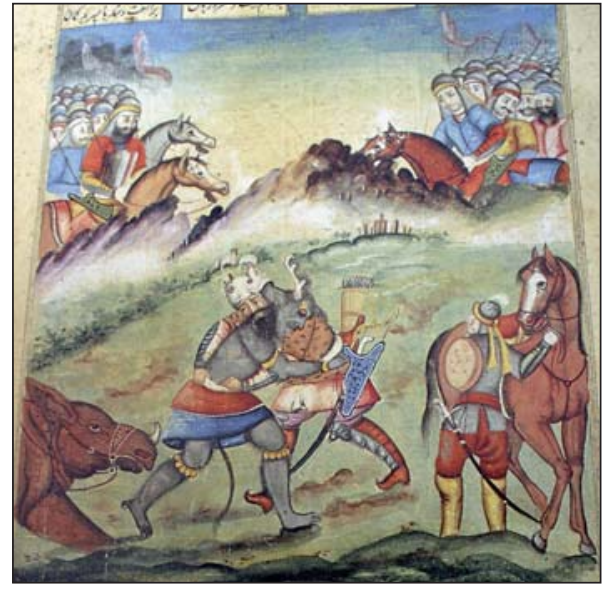

MiNiATURA DE UN MANUSCRITO DEL Š̄̄HNĀME (S. XI DE LA HÉGIRA) TITULADO “LA LUCHA ENTRE ROSTAM Y PULÁDVAND”, EN LA QUE SE APRECIA QUE AMBOS RIVALES SE AGARRAN DEL CINTURÓN Y TRATAN DE DERRIBAR AL OPONENTE.

Cortesía de National Library of Iran.

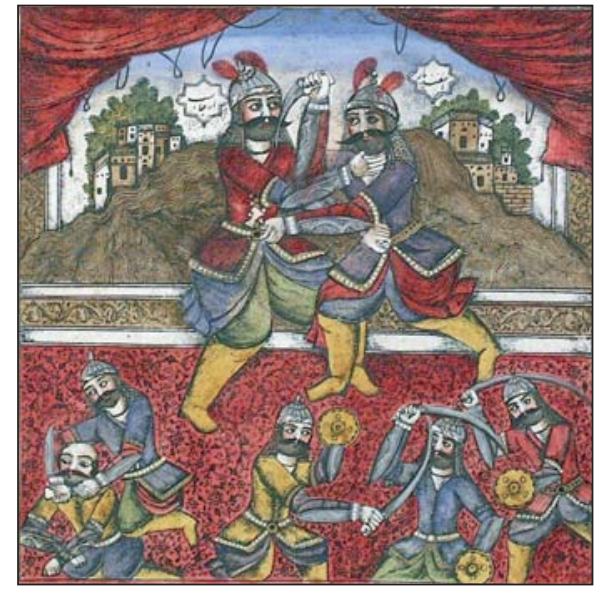

MiNiATURA DE UN MANUSCRITO DEL NEGĀRESTĀN (PERIODO DE QĀJĀR), MUESTRA EL COMBATE ENTRE ARJÁSB Y ESFANDIYÁR EN EL QUE SE APRECIA EL USO DE UNA DAGA Y TÉCNICAS DE LUCHA.

Cortesía de Rezā Abbāsi Museum.

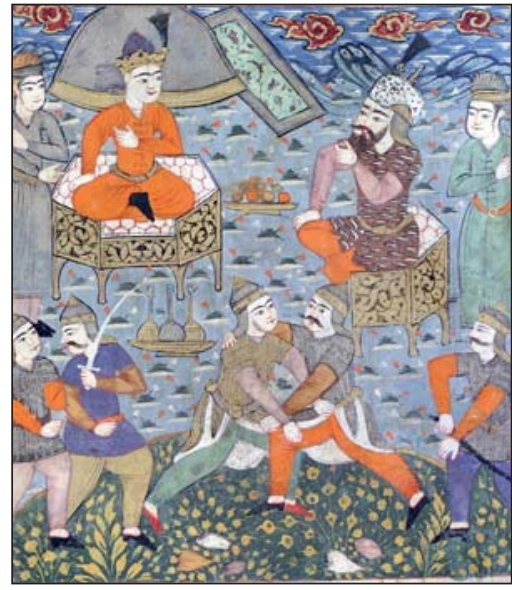

MINIATURA DE UN MANUSCRITO DEL ŠĀHNĀME DEL PERIODO SAFÁVIDA. EL GUERRERO DE LA IZQUIERDA SUJETA EL CINTURÓN Y MUSLO DERECHO DE SU ADVERSARIO PARA LEVANTARLO, MIENTRAS ESTE LE SUJETA LA ESPALDA Y EL MUSLO.

Cortesía de Rezā Abbāsi Museum.

\section{Manuscritos sobre la lucha}

Por fortuna, algunos manuscritos que estudian la lucha con un mayor detalle han resistido el paso del tiempo y han podido llegar hasta nosotros. Hasta donde conocemos, ninguno de estos manuscritos presenta imágenes asociadas a las denominaciones de las técnicas de lucha, lo cual hubiera supuesto una ayuda inestimable para el estudio y comprensión de las mismas. Del mismo modo, y como veremos a continuación, incluso dos de los tres manuscritos más destacados no ofrecen más que las denominaciones de las técnicas, sin profundizar en su descripción.

Uno de los manuscritos más importantes es anónimo y se remonta probablamente al periodo de Šāh Esmāil Safávida (1502-1524 d.C.). Este manuscrito está en la Biblioteca Nacional de Mālek, en Teherán (Beyzāi Kāšāni, 2003/1382: 115-119). El capítulo 13 desarrolla temas sobre el tiro con arco, lucha, combate con lanza, equitación, afilar la espada y similares.

En relación a la lucha, el manuscrito describe 43 técnicas en detalle, con nombres tan curiosos como tariq (camino), qatān (polvo), kaj (torcido) o dastganje (mano del tesoro). Lógicamente, las limitaciones de extensión de este trabajo no nos permiten realizar una descripción detallada de cada una de estas técnicas; no obstante, hay que destacar que algunas de las técnicas citadas en el manuscrito aún se pueden encontrar en las formas de lucha tradicionales iraníes y también en la modalidad de lucha libre olímpica, como describiremos más adelante. 
Otro manuscrito persa dedicado a las técnicas de lucha es el llamado Tumār-e Puryā-ye Vali (El rollo de Puryā-ye Vali), muy probablamente del periodo safávida (1502-1722 d.C.). Puryā-ye Vali era un campeón persa, famoso no solamente por sus habilidades en la lucha sino también por observar escrupulosamente los principios del javānmardi (código ético de los caballeros persas). Este rollo tiene una longitud de 3,5 m y una anchura de $17 \mathrm{~cm}$. El principio del documento está incompleto, y de otra sección se conservan únicamente algunas partes que parecen ser la genealogía de Puryā-ye Vali.

Respecto a la lucha, el manuscrito no ofrece explicaciones específicas sino que únicamente menciona el nombre de las técnicas. También hay que señalar que el autor comete algunos errores en diversas partes del texto; así por ejemplo según el manuscrito se denomina xiz al estudiante de lucha, cuando la denominación correcta es pasxiz. Del mismo modo cita 47 técnicas de lucha, pero repite dos veces la técnica gorg (lobo), y comete errores al enumerar a los estudiantes de lucha. Por desgracia, la aplicación de la mayoria de las técnicas citadas en este manuscrito no está clara, ya que como hemos señalado el texto no ofrece explicaciones sobre las mismas.

Por último, un tercer manuscrito importante que habla de las técnicas de la lucha es Masnavi-ye Golkošti-ye Mirnejāt (Colección de poemas sobre la lucha de Mirnejāt), escrito por Mir Abodola'āl (conocido como Mirnejāt) a finales del periodo de Šāh Soleymān Safávida (1666-1694 d.C.) o a principios del periodo de Šāh Soltān Hosseyn Safávida (1694-1722 d.C.). Este manuscrito es una poesía en forma de masnavi (dísticos rimados).

Mirnejāt fue hijo de un famoso contable del periodo de Šāh Soleymān. Escritor y contable de la oficina real durante el periodo de Šāh Soltān Hosseyn Safávida, fue también bibliotecario de la biblioteca real. Como poeta presentó sus obras en numerosas ocasiones ante el Šāh Soleymān, ganando numerosas recompensas. Una de las características de las poesías de Mirnejāt era su dominio de las palabras utilizadas por el pueblo.

El manuscrito que nos ocupa se compone de 268 rimas, y como bien señala Beyzāi Kāšāni (2003/1382: 393-396), es evidente que Mirnejāt era un buen conocedor de la lucha. Por desgracia, y al igual que sucedía con el anterior manuscrito que hemos referido, Mirnejāt no ofrece explicaciones de las técnicas, sino únicamente sus denominaciones.

\section{Las técnicas de los manuscritos en las formas de lucha actuales en Irán}

La lucha a brazo partido sigue siendo una práctica muy habitual en la sociedad iraní actual. En la región de Xorāsān, al noreste del país, se practica un tipo de lucha tradicional denominada košti-ye bāčuxe. La košti-ye bāčuxe se realiza con una chaqueta de lana sin mangas y un pantalón hasta las rodillas (Qezel Āyāq, 2000/1379), y su desarrollo es similar al del jiujitsu y judo (Mohammad Jahāni, 1998/1377). En el norte de Irán, en la provincia de Gilān, se practica un estilo de lucha tradicional que permite golpes, como puñetazos y patadas, denominado košti-ye gilmardi. Se trata de un arte de combate muy duro porque los luchadores no llevan guantes o protecciones, sino que únicamente visten un pantalón (Qezel Āyāq, 2000/1379:597). El objetivo de esta forma de lucha es derribar al suelo al oponente, pero cuando estos se encuentran a una distancia 
mayor ejecutan potentes golpes con las extremidades superiores e inferiores contra la cara, cabeza, tronco y piernas del rival.

Prácticamente, cada región de Irán tiene su propia forma de lucha que aún se conserva. Además existe la lucha de košti-ye pahlavāni, que se practica en el zurxāne (casa de fuerza) como parte de varzeš-e pahlavāni (literalmente "deporte de campeones", arte marcial tradicional iraní que se practica con instrumentos similares a armas y donde también se practica la lucha a brazo partido). Por ello la lucha libre olímpica es un deporte nacional en Irán y los luchadores iraníes se encuentran entre los mejores del mundo. La mayor parte de estos campeones tienen experiencia en diferentes formas tradicionales de lucha.

Algunas de las técnicas que citan o describen los manuscritos están presentes en las formas de lucha mencionadas. Puesto que dichas formas de lucha se han mantenido en el tiempo desde hace siglos, se puede especular con un alto grado de verosimilitud que estas técnicas se han trasmitido oral, visual y corporalmente de generación en generación, lo cual las convierte en un patrimonio cultural de innegable valor.

Entre las técnicas citadas en el primero de los manuscritos que podemos encontrar hoy en día figuran tres técnicas similares: yānbāši (jefe del costado), yānbāši-e andaruni (jefe del costado interno) y yābāši-ye havā'i (jefe del costado en el aire). En la técnica de yānbāši ambos oponentes están de pie con la pierna derecha adelantada, hombro contra hombro ${ }^{3}$. El luchador sujeta con su axila y mano izquierda el brazo derecho del oponente. A continuación gira hacia la izquierda, pasando su brazo derecho por encima de la cabeza del oponente y sujetando entonces su cuello. Al mismo tiempo, sitúa su mano izquierda en la parte externa de la rodilla derecha del oponente, para por último lograr la proyección enganchando la pierna izquierda del adversario con la pierna derecha y empujando hacia atrás (Rāygān Tafreši, 2007/1386:712; Bolur, 1975/1354: 139). Otra técnica de pie muy habitual en la lucha de varzeš pahlavāni actual (Rāygān Tafreši, 2007/1386: 700; Bolur, 1975/1354: 153), y que también refiere el manuscrito, es mašk-e saqqā (recipiente de aguador), en la que uno de los luchadores gana la espalda al rival y le proyecta cargándole sobre la cadera.

Una técnica, en este caso de suelo, que también existe en la lucha libre tradicional persa y también en la lucha libre olímpica es band-e oštor (ata-camello). En ella uno de los luchadores está en el suelo en cuadrupedia, y el otro luchador detrás de él. Moviéndose hacia su izquierda, atrapa el brazo izquierdo del oponente con su brazo y axila izquierda. Seguidamente pasa la pierna izquierda hacia el lado derecho del oponente, hasta que su cabeza queda controlada por dicha pierna izquierda. Para terminar, empuja con ambos brazos y el resto del cuerpo hacia el costado derecho del oponente hasta lograr girarlo.

En relación a las técnicas citadas en el segundo de los manuscritos, Tumār-e Puryā-ye Vali, podemos citar las de la familia leng (pierna) -leng-e kamar (pierna sobre espalda), leng-e andar ān šāx (pierna entre cabeza), leng-e sarkeš (pierna tira-cabeza), leng-e bahman (pierna de avalancha) y leng-e zangi (pierna negra)-, tangšekar (estrecho de azúcar, también se refiere a la boca de la amante), gorg (lobo), čarxdulāb (rueda de fuente), y kafšak va tang dar amal (zapatito y estrecho en acción), aunque este última técnica está clara sólo par-

3 En todas las técnicas describiremos únicamente la ejecución por un lado. 


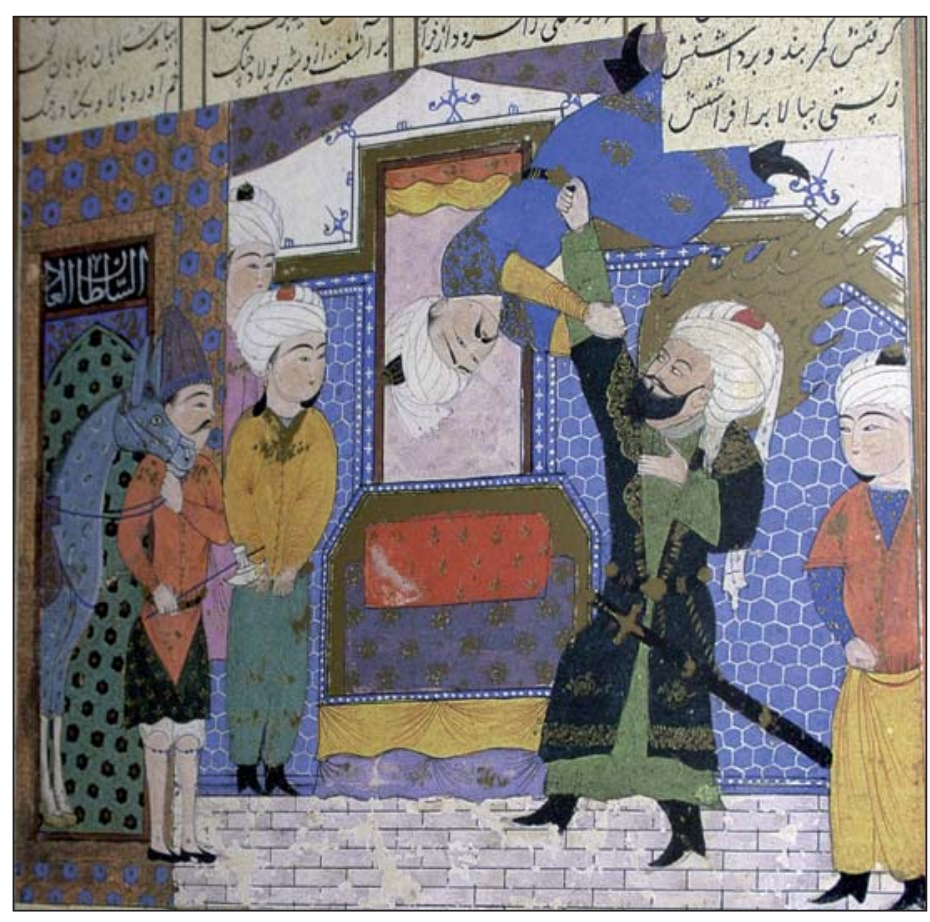

ESCENA DE UN MANUSCRITO DEL X'̄ VARĀNNĀME (S. XV), TITULADA

"EMĀM ALI LUCHA EN LA CORTE DE TAHMĀS", MUESTRA CóMO EMĀM ALI LEVANTA A SU OPONENTE.

Cortesía de Ministry of Culture and Islamic Guidance.

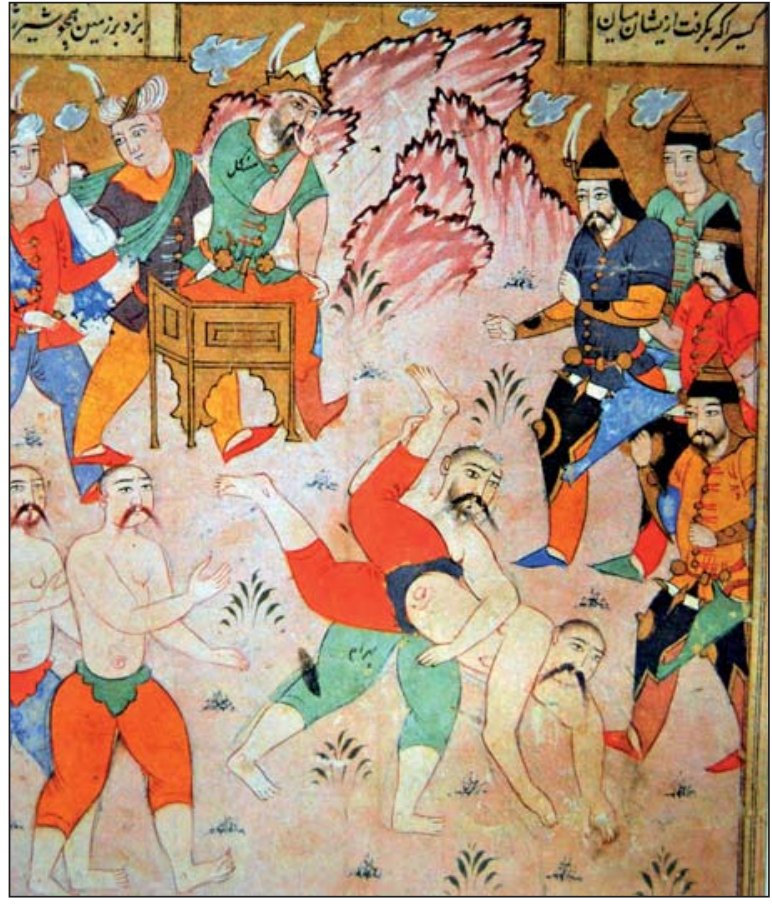

Miniatura “LUChA de BAHRĀM CON EL PAHLAVĀN DE ŠANKAL ŠĀH”, DE UN MANUSCRITO DEL ŠĀHNĀME DATADO EN EL 1048 DE LA HÉGIRA (1638 D.C.), MUESTRA LA APLICACIÓN DE UNA TÉCNICA DE LUCHA.

Cortesía de Golestān Palace Museum.

cialmente. A continuación describiremos brevemente algunas de estas técnicas, junto con algunos problemas que plantean para su asociación con las técnicas descritas en el manuscrito.

Segun Rāygān Tafreši (2007/1386), las técnicas de leng son muy frecuentes en las luchas tradicionales de Irán -el autor cita hasta 21 variantes-, especialmente en las luchas de košti-ye bāčuxe y košti-ye torkaman (otra forma de lucha tradicional de Xorāsān en la que se lucha con una chaqueta). La forma leng-e kamar, por ejemplo, consiste en sujetar estrechamente el brazo izquierdo del oponente bajo la axila derecha, de modo que este quede sobre la espalda. En este momento el oponente tiene su pierna izquierda adelantada, mientras que el luchador que quiere ejecutar la técnica adelanta la derecha y, mientras gira hacia su izquierda, la introduce entre los muslos del rival. El luchador sigue girando y elevando su pierna derecha hasta que proyecta al adversario, que cae al suelo sobre la espalda o costado. En la lucha libre (tradicional y olímpica) esta técnica recibe la denominación leng kardan (hacer pierna).

En el estudio de la técnica tangšekar encontramos mayores dificultades. Según el Lexicon de Ānānderāj consiste en sujetar con las manos fuertemente las piernas del oponente para proyectarlo. No obstante, Rāygān Tafreši (2007/1386: 225) la describe como una forma de proyectar al oponente hacia atrás, colocándose a su costado y bloqueando por detrás sus piernas con el muslo mientras se empuja en esa dirección. A mayores, Bolur (1975/1354: 131) la describe de un 
modo muy diferente, como una técnica de suelo; todo ello nos hace ver que diversas técnicas han podido tener una misma denominación, por lo que a falta de una descripción en los manuscritos no podemos saber a qué técnica en concreto podría referirse el autor del mismo.

También parece claro que ha existido una evolución de algunas técnicas, por ejemplo para atender a nuevas sensibilidades o a cambios en las normas del enfrentamiento. Tal es el caso de la técnica gorg (lobo), que actualmente se realiza en una forma más suave denominada kafgorgi. Originalmente, esta técnica consistía en golpear fuertemente con la palma de la mano diferentes partes del cuerpo del rival, especialmente la cara. Hoy en día este acto está prohibido, pero se ejecuta en forma de empujón con la palma de la mano u hombro sobre la frente del oponente, intentando distraerlo para sujetar sus piernas con las manos y proyectarlo sobre el suelo (Bolur, 1975/1354: 102).

Entre las técnicas citadas en el tercer manuscrito, el Masnavi-ye Golkošti-ye Mirnejāt de Mir Abodola'āl, siguen siendo conocidas dom-e gāv (cola de vaca), gusfandandāz (salta-oveja), kalāte (castillo), meqrāzak (tijera/tijerita), piltepič (girando la cerilla), pišqabz (parte frontal del cinturón), širqalt (rueda-león), xāk (suelo), xeyme zadan (hacer carpa), gāzoravār (como cabra), así como otras ya apuntadas anteriormente como kallekub, kafšak, leng-e kamar, mašk-e saqqā y tangšekar.

La técnica de dom-e gāv existe, pero se considera prohibida (Rāygān Tafreši, 2007/1386: 441) al ser una forma de luxación de rodilla. Como ejemplo de técnicas que se realizan de pie, gusfandandāz se denomina hoy en día dar māye-e dast tu konde-ye gusfandandāz (principio de la mano en el muslo de salta-oveja). En esta técnica ambos luchadores tienen la pierna derecha adelantada. Uno de ellos sujeta la muñeca y brazo derecho del rival con la mano izquierda y derecha respectivamente. Una vez bloqueado el lado derecho del rival, le gana dicho costado girando hacia la izquierda, a la vez que cambia la mano derecha a la nuca del rival, girando y proyectándole hacia el suelo (Rāygān Tafreši, 2007/1386: 317). Otras variaciones actuales de esta técnica son konde-ye gusfandandāz-e darru [tronco de salta-oveja encima] y konde-ye gusfandandāz dar xāk [tronco salta-oveja en el suelo] (Rāygān Tafreši, 2007/1386: 653-654).

Como ejemplo de técnicas en el suelo, kalāte parte con uno de los luchadores en cuadrupedia y otro a su costado izquierdo, controlando la movilidad
MiNIATURA DE UN MANUSCRITO DEL ŠĀHNĀME DATADO EN EL 853 DE LA HÉGIRA (1449 D.C.), EN LA QUE SE MUESTRA UN COMBATE A CORTA DISTANCIA UTILIZANDO LA DAGA Y UNA TÉCNICA DE LUCHA.

Cortesía de Rezā Abbāsi Museum.

MiNIATURA DE UN MANUSCRITO DEL ŠĀHNĀME DATADO EN EL 853 DE LA HÉGIRA (1449 D.C.), EN LA QUE UN GUERRERO CORTA EL CUELLO DE SU ADVERSARIO CON UN CUCHILLO (KĀRD), AL MISMO TIEMPO QUE LE CONTROLA CON UNA TÉCNICA DE LUCHA.

Cortesía de Rezā Abbāsi Museum.

ESCENA DE BATALLA DE UN MANUSCRITO

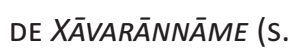
$X V)$, TITULADA “UMAR UMMAYYAH'S PELEA FRENTE A EMĀM ALI”, DONDE UMAR ESTRANGULA A SU OPONENTE.

Cortesía de Ministry of Culture and Islamic Guidance. 


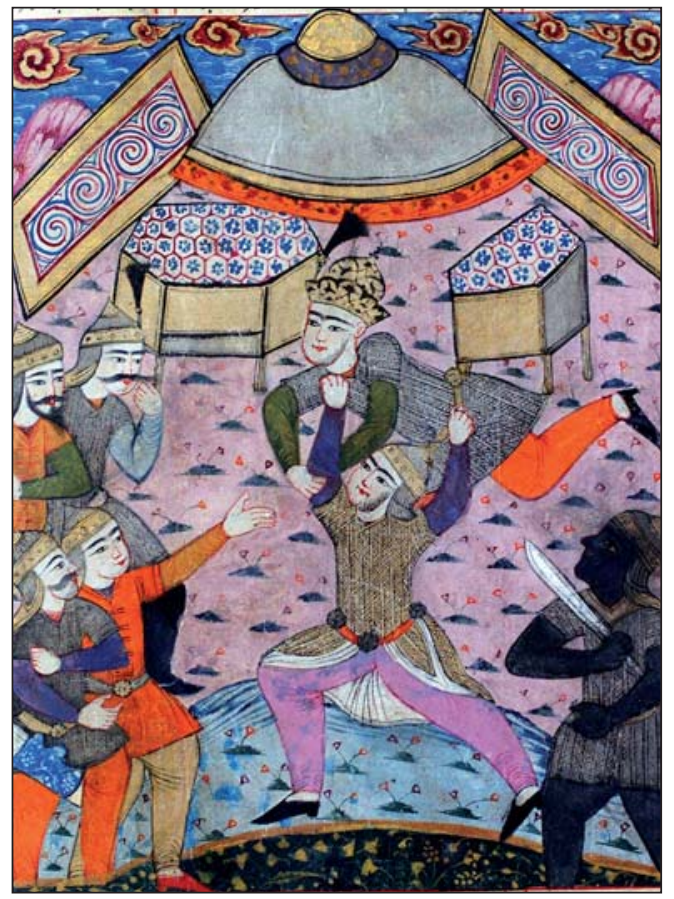

del primero con ambos brazos. Seguidamente pasa el brazo derecho bajo la axila derecha del rival, juntando ambas manos a modo de cierre o candado. Haciendo fuerza con ambos brazos y con las piernas, que han enganchado al rival por la cintura, logra girar al oponente (Rāygān Tafreši, 2007/1386:599). Actualmente existen dos variaciones de esta técnica denominadas kalāteye pišro (castillo adelante) y kalāte be yekpā (castillo con una pierna).

\section{Conclusión}

Como muestran las fuentes históricas, la lucha en Irán jugó un papel muy importante en las batallas, no sólo en los duelos sin armas que se realizaban entre los campeones previamente a su inicio, sino también en combinación con la lucha armada. Hasta la fecha conocemos tres manuscritos sobre la lucha. El primero de ellos, anónimo del periodo de Šāh Esmāil Safávida (1502-1524 d.C.), describe detalladamente 43 técnicas. El segundo, el Tumār-e Puryā-ye Vali (El rollo de Puryā-ye Vali), es también anónimo y data probablamente del periodo safávida (1502-1722 d.C.). Este manuscrito solamente menciona los nombres de 47 técnicas de lucha (46 en realidad, puesto que

MINIATURA DE UN MANUSCRITO DEL ŠĀHNĀME

DEL PERIODO SAFÁVIDA, MUESTRA LA APLICACIÓN DE UNA TÉCNICA DE LA LUCHA PARA LEVANTAR EL OPONENTE.

Cortesía de Rezā Abbāsi Museum una de ellas está repetida), sin describir su ejecución. El tercer manuscrito es el Masnavi-ye Golkošti-ye Mirnejāt, escrito en verso por Mirnejāt a finales del periodo de Šāh Soleymān Safávida (1666-1694 d.C.) o principios del periodo de Šāh Soltān Hosseyn Safávida (1694-1722 d.C.). Aquí Mirnejāt menciona 62 técnicas de lucha, aunque tampoco detalla su ejecución. Afortunadamente, algunas de estas técnicas han sobrevivido en la lucha libre y luchas tradicionales de Irán. Además, en los lexicones persas de diferentes periodos puede encontrarse la descripción de algunas técnicas.

En conjunto, la suma de las técnicas de estos tres manuscritos asciende a 152 técnicas, que se realizaban tanto de pie como en el suelo. En el Golestān (Sa'di, 2005/1383:32) se señala que existían 360 técnicas en la lucha persa, de modo que son muchas las que faltarían en estos documentos. Algunas de ellas, de modo aislado, pueden encontrarse en otros manuscritos. En un notable trabajo, Rāygān Tafreši (2007/1386) presenta 606 técnicas propias de la lucha libre y lucha greco-romana, incluyendo ataques y contrataques en pie y en el suelo. Algunas de estas técnicas son también características de las luchas tradicionales de Irán, mientras que otras están hoy en día prohibidas. También cabe especular con que muchas de las técnicas citadas en los manuscritos persas hayan cambiado de denominación con el transcurso de los siglos. Esperamos que futuras investigaciones sobre los manuscritos persas puedan revelar más nombres y aplicaciones de las técnicas propias de la lucha persa.

\section{REFERENCIAS}

\section{Primarias}

Āsef, Mohammad Hāšem (Rostam al Hokamā) (2003/1382). Rostam al Tavārix: Salatin-e Selseleye Safaviye, Afšāriye, Zandiye va Qājāriye. [Rostam al 
Tavārix: Los reyes de Safávida, Afšārid, Zand y Qājār]. Comentado por Azizollāh Alizādeh. Tehrān: Entešārāt-e Ferdŏs.

Afif, Šamseldin ibn Sarāj ibn (2007/1385). Tārix-e Firuzšāhi [La historia de Firuzšāhi]. Corregido por Velāyat Hosseyn. Introducción por Dr. Mohammad Reza Nasiri. Tehrān: Entešārāt-e Asātir.

Al-Kāteb al-Rajāni, Farāmarz ben Xodādād ben Abdollāh (2004/1383). Samak Ayyār [Samak Ayyār]. Comentado por Seyyed Ali Šahāri. 2 vols. Tehrān: Sedāy-e Moāsser.

Beyqami, Molānā Mohammad (2002/1381). Dārābnāme [La letra/libro de Dārāb]. Corregido y comentado por Zabiollāh Safā. 2 vols. Tehrān: Šerkat-e Entešārāte Elmi va Farhangi.

Beyzāi Kāšānii, Hosseyn Parto (2003/1382). Tārix-e Varzeš-e Bāstāni Iran: Zurxāne [La historia de Varzeš-e Bāstāni-ye de Irān: Casa de fuerza]. Tehrān: Entešārāt-e Zavvār.

Dāstān-e Hosseyn Kord-e Šabestari (2003/1382). The Story of Hosseyn Kord Šabestari. Comentado por Abbās Šabgāhi Šabestari. Tehrān: Moaseseye Entešārāt-e Farahāni.

Daqiqi Tusi, Abu Mansur Mohammad ebn-e Ahmad (1994/1373). Goštāsbnāme be Enzemām-e Farhang-e Basāmadi [Letra de Goštāsb junto con el Basāmadi Lexicon]. Comentado por Mohammad Javād Šari'at. Tehrān: Entešārāt Asātid.

Ferdŏsi, Hakim Abolqāsem (1995/1384). Šāhnāme Ferdŏsi (bar Asās-e Nosxeye Mašhur-e be Čap-pe Mosko) [Letra/libro de reyes de Ferdŏsi (basado en la versión de un manuscrito publicado en Moscú)]. Tehrān: Entešārāt-e Alam.

Hakim, Manouchehr Xān (s.f.). Koliyat-e Haft Jeldi Eskandarnāme: Sahebqarān Zowalgarenein Eskandar ben Dārāb ben Bahman ben Esfandiār Ruintan [Los siete volúmenes completos de la letra/libro de Eskandar: Sahebqarān Zowalgarenein Alexander, el hijo de Dārāb, el hijo de Bahman, el hijo de Invulnerable Esfandiār]. De la serie de leyendas de Pahlavān oriental. Tehrān: Entešārāt-e Mohammad Hasan Sar Elmi.

Kāzeruni, Jafar (1997/1376). Tārix-e Pahlavāni Dar Kermanshah - Dar Do Qarn Axir (1174-1374 Hégira Šamsi) [La historia de Pahlavāni en Kermānšāh - en los dos siglos]. 2 vols. Kermānšāh: Entešārāt Tāqe Bostān.

Mobārak Šāh Faxr-e Modabbar, Mohammad ben Mansur ben Said (1967/1346). $\bar{A} d \bar{a} b$ al-Harb va al- al-Šojā-e [Costumbres de la guerra y el coraje]. Comentado por Ahmad Soheili Xānsari. Tehrān: Eqbāl.

Molavi, Mŏlānā Jalāl-ad-din Mohommad Rumi (2007/1385). Masnavi Ma'navi [EI Verso que rima con el que le sigue con un sentido profundo]. Comentado por Reynold A. Nicholson. Tehrān: Entešārāt Hermes.

Qatrān-e Tabrizi, Abu-Mansur (1983/1362). Divān-e Qatrān-e Tabrizi [Poemas de Qatrān-e Tabrizi]. Basado en un manuscrito de Mohammad Naxjavāni. Tehrān: Entešārāt-e Qoqnŏs.

Rašidoldin Fazlollāh Hamedāni, Al Vazir Ibn Emadolddole Abi al-Xeir Movafag al-Dole Ali (1985/1374). Jāme-al Tavārix (Az Aqāz Peidayeš Moqol Ta Pāyān Doreye Teimur Qā'ān) [La historia completa (desde la emergencia de los mongoles hasta el final de la era de Timur Qā'an)]. Escrita en el 703 de la Hégira (1304 d.C.). Comentada por Bahman Karimi. Tehrān: Entešarat Eqbāl. 
Romuz-e Hamze [Secretos de Hamze] (1940/1359 de la Hégira). Manuscrito por Mohammad Ali Nāmē. Tehrān: Šerkat-e Čāp-e Ketāb.

Sa'di, Moslehodin Mošref ben Abdollāh (2005/1383). Koliyāt-e Sa'di [Obras completas de Sa'di]. Comentado por Hasan Anvari. Tehrān: Našr-e Qatre.

Šabānkāre'i, Mohammad Ben Ali Ben Mohammad (2002/1381). Majma' alAnsāb (Nimeye Avval) [Majma' al-Ansāb (Primera Parte)]. Comentado por Mir Hāšem Mohaddes. Tehrān: Amir Kabir.

Širuye Nāmdār (2005/1384). Tehrān: Entešārāt-e Qoqnŏs.

Xonji Esfahāni, Fazlallāh ibn Ruzbehān (2003/1382). Tārix-e Ālam Āryā-ye Amini: Šarh-e Hokmrāni Salātin-e Āq- Quyunlu va Zohur-e Safaviān [La historia de Ālam Āryā-ye Amini: La exposición de sultanes de Aq-quyunlu's soberanos hasta la aparición de safávidas]. Editado por Mohammad Akbar Ashiq. Tehrān: Markaz-e Nashr Miras Maktub.

\section{Secundarias}

Abbāsi, Mehdi (1995/1374). Tārix-e Koshti-ye Irān [Historia de lucha de Irān]. Vol. 1. Tehrān: Entešārāt-e Ferdǒs.

Afshari, Mehran (2003/1382). Fotovvatnāmehā va Resāle-ye Xāksariye (Si Resāle) [Libros de la caballería y el manuscrito de Xāksariye: treinta manuscritos]. Tehrān: Pajuhešgāh-e Olum-e Ensāni va Motāleāt-e Farhangi.

Aštari Tafreši, Ezatollāh (2000/1379). Nehzat-e Ayyārān az Aqāz Tā Dore-ye Pahlavi [El movimiento de Ayyārān desde el principio hasta el periodo de Pahlavi]. Tehrān: Šarat Āvāye Nur.

Bolur, Habibollāh (1975/1354). Fan va Band-e Košti [Técnicas de lucha]. Tehrān: Entešārāt-e Madrese-ye Āli-e Varzeš.

Farahvaši, Bahrām (2002a/1381). Farhang Zaban Pahlavi [Lexicón de la lengua pahlavi]. Tehrān: Entešārāt-e Danešgāh-e Tehrān.

Farahvaši, Bahrām (2002b/1381). Farhang Farsi be Pahlavi [Lexicón de la lengua persa en pahlavi]. Tehrān: Entešārāt-e Dānešgāh Tehrān.

Jacob, Alain (1985). Les Armes Blanches du Monde Islamique: Armes de Poing: Épées, Sabres, Poignards, Couteaux. Paris: Jacques Grancher.

Kāzemini, Kāzem (1964/1343). Naqš-e Pahlavāni Va Nehzat-e Ayyāri Dar Tārix-e Ejtemāi Va Hayāt Siyāsi-ye Melat-e Irān [El rollo de Pahlavānān y el movimiento de Ayyāri en la historia social y política de Irán]. Tehrān: Čāpxāne-ye Bānk Melli Irān.

Mohammad Jahāni, Ali (1988/1377). Košti Kordi ba Čuxe [La lucha kurda de bā Čuxe]. Tehrān: Našre Beh Āfarin.

Qezel Āyāg, Soraya (2000/1379). Rāhnamā-ye Bāzihā-ye Irān [Guía de los juegos de Irán]. Tehran: Daftar Pajuheshhaye Farhangi.

Rāygān Tafreši, Abolqāsem (2007/1386). Ganjine-ye Fonun-e Āzād va Farangi [El tesoro de las técnicas de la lucha libre y grecoromana]. Tehrān: Entešārāt-e Safir Ardahāl.

Zakeri, Mohsen (1995). Sasanid Soldiers in Early Muslim Society: The Origins of Ayyaran and Futuvva. Wiesbaden: Harrassowitz Verlag. 